\title{
Introduction: special issue JIMB-BioMicroWorld 2009
}

\author{
James Gomes
}

(C) Society for Industrial Microbiology 2010

In its brief history, the BioMicroWorld conference has grown in stature and is today recognized as one of the premier international conferences that promotes applied research in microbiology and microbial biotechnology across disciplines. Its III International Conference on Environmental, Industrial, and Applied Microbiology (BioMicroWorld 2009) was held in Lisbon, Portugal, on December 2-4, 2009. The conference brought together engineers, scientists, and researchers in the fields of industrial microbiology, biotechnology, biological sciences, biochemical engineering, biodegradation, environmental sciences, and other related fields, to present their research and discuss the challenges in the theme areas of the conference. In total, 600 participants from 54 countries attended BioMicroWorld 2009. This special issue of the Journal of Industrial Microbiology and Biotechnology contains a compilation of peer-reviewed papers from the conference.

Each day of the conference began with a plenary lecture by a renowned researcher followed by oral presentations from speakers around the world. The conference was organized into five main sessions covering the following topics:

(1) Environmental microbiology, geomicrobiology; (2) Agriculture, soil, forest microbiology; (3) Food microbiology; (4) Industrial microbiology-future bioindustries; (5) Methods-analytical and imaging techniques; (6) Medical and pharmaceutical microbiology, antimicrobial agents; (7) Microbial physiology, metabolism and gene

J. Gomes $(\bowtie)$

School of Biological Sciences, Indian Institute of Technology

Delhi, Block 1-A, Room 307, Hauz Khas,

New Delhi 110016, India

e-mail: jgomes@bioschool.iitd.ac.in expression; (8) Biofilms and antimicrobial surfaces; (9) Bioremediation; (10) Biotechnologically relevant enzymes and proteins; (11) Microfactories - microbial production of chemicals and pharmaceuticals.

Three plenary lectures were delivered at this conference. The first plenary lecture was delivered by Prof. Hermann Heipieper from the Helmholtz Centre for Environmental Research-UFZ, Germany. He delivered an inspiring lecture entitled "Microbial adaptation to toxic organic solvents-mechanisms and biotechnological applications" in which he presented the physiology and biochemistry of solvent-tolerant bacteria and their specific adaptive mechanisms. He discussed the toxicity of fine chemicals in relation to hydrophobicity of biological membranes. In this context, he examined why bacteria adapted to these chemicals were powerful biotransformants, having potential application in bioremediation and biocatalysis. In the second plenary lecture, Prof. Stefan Dubel from the Institute for Biochemistry and Biotechnology, Technical University of Braunschweig, Germany, expounded on "Antibodies from bacteria". He discussed the challenges facing the functional decryption of the human proteome. He stated that in vitro, antibody selection with antibody phage display offers a solution that can meet the huge demand of more than 25,000 antibodies. He presented the intricacies in creating antibody gene libraries and constructing high-throughput pipelines for this purpose. Prof. Rosario de Oliviera from the Department of Biological Engineering, University of Minho, Portugal gave the third plenary lecture on "Insights into the biofilm world". In this lecture, Prof. Oliviera gave her insight on why it was so difficult to eradicate harmful biofilms. She examined the physiology of sessile cells and its relation to the recalcitrance observed in biofilms. The higher incidence of occurrence of medical biofilms in catheters, prostheses, and 
other medical implants, and the increase of microorganisms' resistance to antimicrobial agents was presented as one of the major concerns in health science.

The journal is proud to bring to its readers a bouquet of 30 original papers selected from the presentations made at the different sessions of the conference. These papers have been accepted after a rigorous peer-review process. This has ensured that the papers are within the scope of the Journal of Industrial Microbiology and Biotechnology and meets its standards. In addition, the papers are highly relevant and representative of the status of research in the theme areas of the conference. The papers can be broadly classified into the following areas: environmental microbiology, biopesticides, fermentation, food and wine microbiology, and medical microbiology. This heterogeneous ensemble will give the reader a flavor of the conference. The original papers collected in this special issue reflect a high standard of academic and industrial research. I hope that readers find the contents of this issue interesting and valuable.

On behalf of BioMicroWorld, I would like to thank all members of the organizing and Scientific Advisory Committees for their efforts to make the conference successful. I would also like to thank all the delegates of the conference and the authors for sharing their valuable research with the community at large, and the referees for sparing their valuable time to review the papers. Thanks are also due to the Editor-in-Chief Dr. Allen I. Laskin and his editorial team whose enormous effort has made it possible to publish this edition of the special issue. Finally, I express my gratitude to BioMicroWorld for giving me this opportunity. 